\title{
Resistance Training Effects on Arterial Compliance in Premenopausal Women
}

\author{
Anette S. Fjeldstad, PhD, Michael G. Bemben, PhD, and \\ Debra A. Bemben, PhD
}

Endurance training has been shown to increase arterial compliance; however, the effect of resistance training is unclear. Purpose: The purpose of this study was to examine the effect lower body resistance training on arterial compliance in healthy premenopausal women. Methods: Thirty-two women were assigned to a resistance training group $(n=21)$ or a control group $(n=$ 11). Large (C1) and small (C2) arterial compliance (Pulse Contour Analysis) were measured at baseline and after twelve weeks of training. Results: Two-way (group $\times$ time) repeated measured ANOVA did not detect significant group, time effects or group $\times$ time interactions for small arterial compliance $(P>0.05)$. There was a significant time effect for large arterial compliance $(P<0.05)$, which increased in both groups. Conclusions: In contrast to previous studies in men, which found decrease in arterial compliance with resistance training, no decrease in arterial compliance was observed.

Keywords: vascular stiffness; lower leg; premenopausal women

\section{Introduction}

Over the last decade, there has been increasing interest in vascular stiffness, the loss of blood vessel wall compliance in the major arteries, because it is a risk factor for cardiovascular events, especially stroke. ${ }^{1}$ Arterial compliance is defined as the ability of an artery to expand and recoil with cardiac pulsation and relaxation. ${ }^{2}$ The cardiovascular structure and function include thickening of the vessel wall, a decrease in systemic arterial compliance, and cross-sectional distensibility of the large elastic arteries. These changes lead to an increased cardiovascular disease risk in elderly participants. ${ }^{3}$

Exercise has shown to be beneficial for protection against atherosclerotic vascular damage in elderly male athletes compared to elderly sedentary males ${ }^{4}$ and to attenuate the age-related changes in

From the Department of Medicine, Division of Geriatrics, University of Utah and Geriatric Research, Education, and Clinic Center (GRECC), Salt Lake City, Utah (ASF); and Department of Health and Exercise Science, University of Oklahoma, Norman, Oklahoma (MGB, DAB).

Address correspondence to: Anette S. Fjeldstad, George E. Whalen VA Medical Center, 500 Foothill Drive, Salt Lake City, UT 84148; e-mail: a.fjeldstad@utah.edu. arterial stiffness in elderly male athletes, ${ }^{5}$ which may reduce the risk of experiencing a cardiovascular event. ${ }^{6,7}$ Habitual exercise has been associated with a decrease in arterial stiffness (increased compliance) in women, and these benefits were associated with lack of increase in systolic and arterial pulse pressures. ${ }^{8}$ Furthermore, Kingwell ${ }^{9}$ showed that aerobically trained middle-aged athletes had lower vascular stiffness than their sedentary counterparts, and time to exhaustion on treadmill testing in older participants correlated positively with arterial compliance.

Both cross-sectional studies ${ }^{10}$ and intervention resistance training studies ${ }^{11}$ have reported decreases in large arterial compliance $(\mathrm{C} 1)$ in healthy men, whereas endurance training has reported to have a positive effect on the vasculature, such as increasing arterial compliance. ${ }^{4,12,13}$ Although aerobic exercise seems to have beneficial effects on the vasculature, this effect is not maintained in the absence of exercise, ${ }^{12}$ suggesting the importance of maintaining a physically active life to sustain a healthy vascular function.

Based on previous literature, it is evident that endurance-type exercise has beneficial effects on the vasculature, in terms of downregulating and 
decreasing various risk factors associated with endothelial dysfunction, such as hypertension, diabetes, obesity, and inflammatory markers. ${ }^{14-16}$ Resistance training has profound effects on the musculoskeletal system, contributing to maintenance of muscle strength and bone health. ${ }^{17}$ However, the effect of resistance training on the cardiovascular system is still unclear, with the majority of investigations completed in men. ${ }^{10,11}$ There are very few studies examining this issue in women, ${ }^{18,19}$ and because of sex-related differences in the vasculature, ${ }^{20}$ the results obtained in men cannot easily be extrapolated to women. The purpose of this study was to examine the effects of resistance training on $\mathrm{C} 1$ and small arterial compliance (C2) in healthy premenopausal women. We hypothesized that arterial compliance would decrease after 12 weeks of resistance training.

\section{Methods}

\section{Participants}

Recruitment. The participants of this study were healthy, premenopausal women between the ages of 19 and 48 years $(n=32)$. All participants were free of signs, symptoms, and history of any overt chronic diseases. None of the participants had a history of smoking, were taking any hormonal medication, antihypertensive drugs, or were diabetic. The participants were leisurely active with no current history of resistance training. The participants also completed a Health History and a Menstrual History Questionnaire. The participants were volunteers from the University of Oklahoma, Norman campus, and the surrounding areas. Participants read and signed a written informed consent as approved by the University of Oklahoma Institutional Review Board.

Research design. This study used a mixed factorial repeated measures design as a nonexercising control group $(\mathrm{C}, \mathrm{n}=11)$ and a training group $(\mathrm{T}, \mathrm{n}=21)$ were assessed before and after the 12-week training period (3 days per-week). The participants were randomly assigned either to a morning session or an afternoon session. Before and after the intervention period, all study participants underwent 1 repetition maximum (1-RM), dual energy X-ray absorptiometry (DXA) total body scans, measures of height and weight, and measures of $\mathrm{C} 1$ and C2. To avoid potential diurnal variations, the participants were tested in a fasted and rested state in the morning of both visits. Using menstrual history questionnaires, attempts were made to study the women in the same phase of their menstrual cycle before and after the intervention.

\section{Measurements}

Resistance training. The resistance training exercises primarily targeted sites at the hip and leg, and lower leg muscle groups. Specific exercises included hip flexion/extension, hip abduction/adduction, supine leg press, calf raises, and shin exercise. The "shin" exercise was performed seated with the top of the foot weighed down while performing dorsiflexion movements of the foot and ankle, thereby targeting the tibialis anterior muscle. The resistance exercises were performed using isotonic weight training equipment (Cybex Inc, Medway, Mass). The 1-RM was obtained by finding the maximum load that the participant could move with good form through the entire range of motion. Two warm-up sets with 8 to 10 repetitions and 3 to 5 repetitions with progressing load were completed before the first 1-RM attempt. The 1-RM was found within 5 attempts, with 1-minute rest between each attempt. The 1-RM testing was supervised and recorded by trained personnel. The participants performed 2 sets of 8 repetitions at $80 \%$ of their 1-RM for all hip exercises. The remaining exercises consisted of 3 sets of 8 repetitions at $80 \%$ of their 1-RM. Each exercise session was completed in about 30 minutes, with a 2 to 3 minutes rest period between each set. Trained personnel supervised all exercise sessions, encouraged participants, and helped record and change the weights whenever necessary. The control group was instructed to maintain their normal level of physical activity for the 12-week intervention period.

Body composition measurements. Dual energy X-ray absorptiometry (GE Lunar Prodigy, enCORE software version 10.50.086, Madison, Wis) was used to determine body composition variables (percentage body fat, fat mass, fat-free mass). Participants removed any metal or plastic before laying down in a supine position on the DXA platform. Participants were instructed to remain still as the scanner arm moved down the body. Quality assurance procedures were performed daily to ensure proper calibration 
prior to scanning. The coefficient of variation for total body bone mineral density using DXA in the Bone Density Research Laboratory is $1 \%$.

Pulse contour analysis. Arterial compliance was measured using the HDI/Pulsewave CR-2000 and the CVProfilor DO-2020 CardioVascular Profiling System (Hypertension Diagnostic, Inc, Eagan, Minn). The device consisted of an oscillometric blood pressure module, a noninvasive Arterial PulseWave Sensor and a 486-75 MHz computer and medical electronics, which included a touch screen and software. The height and weight was measured and entered into the 486-75 MHz computer. The participant rested in a supine position for approximately 10 minutes. An appropriate-sized cuff was wrapped around the participant's upper left arm. A rigid plastic wrist stabilizer was placed on the participant's right wrist to minimize any movement and stabilize the radial artery during the three 30-second collection of blood pressure waveform data. A noninvasive Arterial PulseWave Sensor was positioned on the surface of the skin overlying the right radial artery at the point of the strongest pulsation to capture an analog blood pressure waveform. The sensor was adjusted to the highest relative signal strength, without occluding the artery. This device can measure the decay in diastolic pressure in the central arteries and the decay in the reflective waves of the peripheral arteries. Other cardiovascular parameters that were assessed during the pulse contour analysis (PCA) testing included blood pressure, pulse rate, systemic vascular resistance, and total vascular impedance. Three consecutive trials were performed, and because 1way repeated measures analysis of variance (ANOVA) showed no significant differences between the trials, the trials were averaged for the subsequent analyses.

This noninvasive instrument has proven to be reliable $^{21-24}$ for the measurement of $\mathrm{C} 1$ and $\mathrm{C} 2$, and it is a useful method for assessing atherosclerosis. ${ }^{25}$ The investigator's precision of the PCA measurements was determined by measuring 9 healthy participants on 2 different days, 2 to 3 days apart. The coefficient of variation for $\mathrm{C} 1$ and $\mathrm{C} 2$ measurements obtained by the same operator were $6.7 \%$ and $10.6 \%$, respectively. The intraclass correlation coefficients for $\mathrm{C} 1$ and $\mathrm{C} 2$ were 0.918 and 0.985 , respectively.

\section{Statistical Analysis}

Descriptive statistics are reported in mean \pm standard error (SE) for the dependent variables. The
Table 1. Physical Characteristics at Baseline for Each Group $^{a}$

\begin{tabular}{lrr}
\hline & \multicolumn{2}{c}{ Group } \\
\cline { 2 - 3 } Variable & Training $(\mathrm{n}=21)$ & Control $(\mathrm{n}=11)$ \\
\hline Age (years) & $33.2 \pm 2.1$ & $36.8 \pm 3.2$ \\
Body weight $(\mathrm{kg})$ & $74.2 \pm 4.5$ & $67.0 \pm 3.3$ \\
Height $(\mathrm{cm})$ & $166.1 \pm 1.1$ & $165.6 \pm 2.1$ \\
BMI $\left(\mathrm{kg} / \mathrm{m}^{2}\right)$ & $26.6 \pm 1.4$ & $24.4 \pm 1.1$ \\
Body fat $(\%)$ & $36.5 \pm 1.8$ & $35.7 \pm 3.1$ \\
\hline
\end{tabular}

NOTE: BMI = body mass index.

${ }^{\text {a }}$ Values are means \pm SE.

effects of the training on $\mathrm{C} 1$ and $\mathrm{C} 2$ were analyzed by 2 -way (group $\times$ time) repeated measures ANOVA. Analysis of covariance (ANCOVA) was used to examine $\mathrm{C} 1$ responses after adjusting for body weight, body mass index (BMI), percentage body fat, and age. Based on sample size previously documented in the literature that obtained $>80 \%$ power, a priori calculations revealed adequate sample size in the current study. However, these estimates were based on different techniques and/or populations. The level of significance was set at $P \leq .05$. All statistical procedures were performed by SPSS 11.5 software (Chicago, Ill).

\section{Results}

A total of 32 participants completed the 12 weeks of the study and were included in the analyses. The participants in the intervention group completed $85 \%$ of the scheduled training sessions. Table 1 displays the baseline physical characteristics. There were no significant differences $(P>.05)$ between groups for these variables at baseline.

Cardiovascular responses to training. Table 2 shows the mean baseline and posttest cardiovascular measures for the training and the control groups. Diastolic blood pressure had no significant group, or group $\times$ time interaction effects, but did show a significant time effect $(P \leq .05)$, by decreasing. Systolic blood pressure showed no group or group $\times$ time interaction effects but did show a trend for time effect $(P$ $=.073$ ). Heart rate, pulse pressure, systemic vascular resistance, and total vascular impedance did not significantly $(P>.05)$ change from baseline to the end of the training period. 
Table 2. Cardiovascular Variables ${ }^{\mathrm{a}}$

\begin{tabular}{|c|c|c|c|c|}
\hline \multirow{2}{*}{ Variable } & \multicolumn{4}{|c|}{ Group } \\
\hline & \multicolumn{2}{|c|}{ Training $(\mathrm{n}=21)$} & \multicolumn{2}{|c|}{ Control $(\mathrm{n}=11)$} \\
\hline RHR (beats/min) & $66.3 \pm 1.9$ & $65.2 \pm 2.4$ & $61.7 \pm 2.9$ & $58.9 \pm 3.1$ \\
\hline $\mathrm{SBP}(\mathrm{mm} \mathrm{Hg})$ & $118.9 \pm 2.4$ & $116.2 \pm 3.0$ & $117.9 \pm 3.0$ & $113.6 \pm 2.1$ \\
\hline $\mathrm{PP}(\mathrm{mm} \mathrm{Hg})$ & $49.8 \pm 1.2$ & $49.5 \pm 1.3$ & $48.2 \pm .9$ & $46.1 \pm .8$ \\
\hline $\mathrm{Cl}(\mathrm{mL} / \mathrm{mm} \mathrm{Hg} \times 10)$ & $5.9 \pm .6$ & $17.2 \pm 1.1^{\mathrm{b}}$ & $16.0 \pm .9$ & $18.1 \pm 1.3^{\mathrm{b}}$ \\
\hline $\mathrm{C} 2(\mathrm{~mL} / \mathrm{mm} \mathrm{Hg} \times 100)$ & $7.6 \pm .5$ & $7.8 \pm .6$ & $8.2 \pm .9$ & $8.6 \pm .9$ \\
\hline SVR (dyne $\left.\cdot \mathrm{s} \cdot \mathrm{cm}^{-5}\right)$ & $1263.9 \pm 63.2$ & $1167.4 \pm 47.6$ & $1334.0 \pm 57.2$ & $1284.6 \pm 44.9$ \\
\hline TVI (dyne $\left.\cdot \mathrm{s} \cdot \mathrm{cm}^{-5}\right)$ & $126.5 \pm 6.4$ & $120.2 \pm 5.4$ & $128.4 \pm 5.3$ & $120.5 \pm 3.9$ \\
\hline
\end{tabular}

NOTES: $\mathrm{C} 1=$ large arterial elasticity; $\mathrm{C} 2=$ small arterial elasticity; $\mathrm{DBP}=$ diastolic blood pressure; hypertension $=\mathrm{SBP} / \mathrm{DBP}>130 /$ $85 \mathrm{~mm} \mathrm{Hg} ; \mathrm{PP}=$ pulse pressure; RHR = resting heart rate; $\mathrm{SBP}=$ systolic blood pressure; $\mathrm{SVR}=$ systemic vascular resistance; TVI $=$ total vascular impedance.

${ }^{\text {a }}$ Values are mean \pm SE.

${ }^{\mathrm{b}} P<.05$.

Table 3. Muscular Strength at Baseline for Each Group $^{\mathrm{a}}$

\begin{tabular}{lcc}
\hline & \multicolumn{2}{c}{ Group } \\
\cline { 2 - 3 } Variable & Training $(\mathrm{n}=21)$ & Control $(\mathrm{n}=11)$ \\
\hline Hip flexion $(\mathrm{kg})$ & $48.8 \pm 1.9$ & $51.6 \pm 2.0$ \\
Hip extension $(\mathrm{kg})$ & $67.3 \pm 2.9$ & $72.3 \pm 4.0$ \\
Abduction $(\mathrm{kg})$ & $39.6 \pm 1.2$ & $40.2 \pm 2.0$ \\
Adduction $(\mathrm{kg})$ & $49.2 \pm 1.8$ & $52.4 \pm 2.0$ \\
Leg press $(\mathrm{kg})$ & $107.3 \pm 4.4$ & $101.2 \pm 6.1$ \\
Calf $(\mathrm{kg})$ & $51.6 \pm 2.7$ & $46.2 \pm 3.4$ \\
Shin $(\mathrm{kg})$ & $17.9 \pm 1.1$ & $19.6 \pm 1.3$ \\
\hline
\end{tabular}

${ }^{\text {a }}$ Values are means $\pm \mathrm{SE}$.

Large and small arterial compliance. The results showed that there was no significant group effect or time $\times$ group interaction effect for $\mathrm{C} 1$, but a significant time effect occurred with an increase in $\mathrm{C} 1$ from pretest to posttest. The same analyses for $\mathrm{C} 2$ revealed no significant group, time, or time $\times$ group interaction effects $(P>.05)$.

After adjusting for body weight, BMI, and percentage body fat in separate analyses, there was no longer a significant time effect for $\mathrm{C} 1(P>.05)$. After adjusting for age, there still was a significant time effect for $\mathrm{C} 1$. The unadjusted $\mathrm{C} 1$ and $\mathrm{C} 2$ responses to 12 weeks of training are presented in Table 2 .

Muscular strength responses to training. There were no significant differences $(P>.05)$ between groups at baseline for any of the strength variables (Table 3 ).
The percentage changes in strength after the 12-week intervention period is presented in Figure 1. From baseline, the training group significantly $(P<.05)$ increased $1-\mathrm{RM}$ strength for all exercises after 12 weeks of resistance training compared to the control group. There was a significant increase in strength $(P<.05)$ for hip abduction and the calf exercise in the control group. However, the magnitude of change was significantly smaller compared to that of the intervention group.

\section{Discussion}

We examined the effects of lower body resistance training on $\mathrm{C} 1$ and $\mathrm{C} 2$ in healthy premenopausal women. The uniqueness of this study is the examination of arterial compliance following lower body resistance training in women. It has been well documented in previous research that endurance training increases arterial compliance, ${ }^{4,5,12,13,26}$ whereas the effect of resistance training on arterial compliance is still unclear as contradictory results have been documented..$^{10,11,19,27-32}$ Most of the studies have shown a decrease in arterial compliance following resistance training, ${ }^{10,11,19}$ whereas others have shown no changes, ${ }^{28}$ or even an increase in arterial compliance. ${ }^{33}$

To our knowledge, this is the first study that has examined arterial compliance after 12 weeks of lower body resistance training in women. Interestingly, we did not find the negative effect on the arteries that has been previously reported with resistance training. There are studies that have reported an increase 


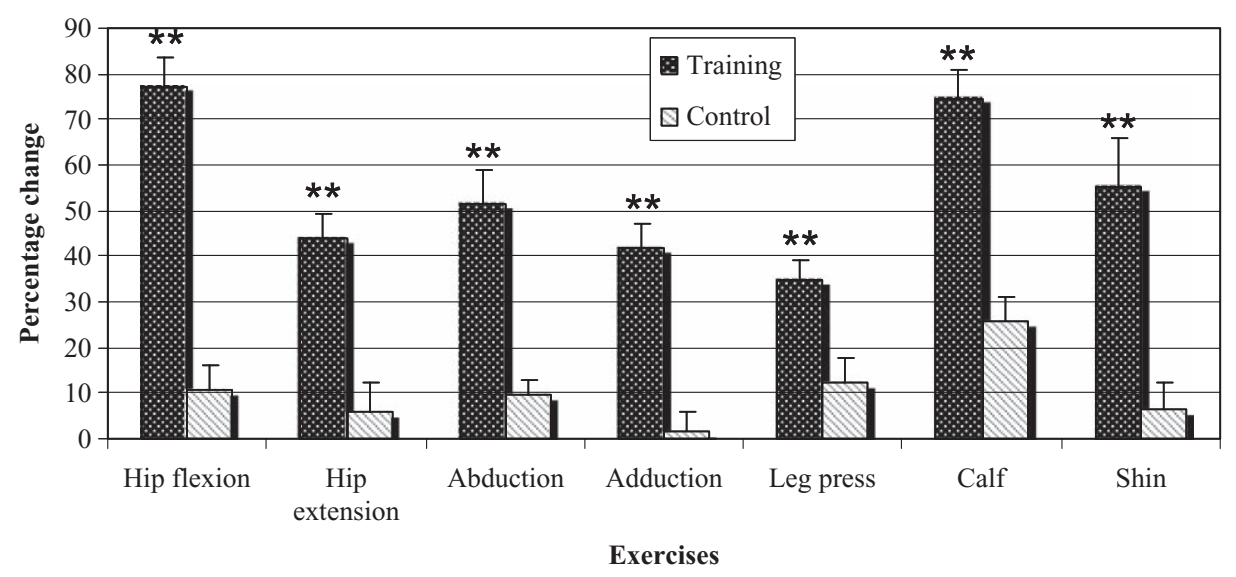

Figure 1.

in arterial stiffness after 4 months of resistance training, ${ }^{11}$ whereas others have found arterial compliance unchanged after 12 weeks of training. ${ }^{28}$ The exact mechanism in which arterial compliance is modified during resistance training is not well understood. It has been suggested that the frequent increase in arterial blood pressure ${ }^{10}$ and shear stress ${ }^{27}$ in the arterial wall during resistance training are contributing factors to the often observed decrease in arterial compliance. Other suggestions are greater sympathetic nerve activity, decreased nitric oxide bioavailability, and increased levels of vasoconstrictor hormones. ${ }^{19}$

This study showed a significant increase over the 12 weeks in $\mathrm{C} 1(P<0.05)$ for both groups. Because this increase occurred in both groups, the changes cannot be attributed to the resistance training. A similar study, which also examined the effects of 12 weeks of lower body resistance training on arterial function, found no change in arterial compliance with training. ${ }^{34}$ This study only examined men, had a relatively small sample size $(\mathrm{n}=11)$, and did not have a control group for comparison. Because the participants only exercised twice per week at $60 \%$ of $1-\mathrm{RM}$, the resistance training program may not have been strenuous enough to elicit a modification in the vasculature. Cortez-Cooper et al ${ }^{19}$ examined arterial stiffness after 11 weeks of whole body high-intensity resistance training in young women. Carotid augmentation index and pulse wave velocity as a measure of large arterial stiffness increased significantly for the training group compared to that of the control group. The discrepancy between our findings and those of Cortez-Cooper et al ${ }^{19}$ may be explained in part to training program differences as our protocol was less strenuous and focused solely on lower body resistance exercises versus CortezCooper's protocol that included whole body, high-intensity strength, and power training. This suggests that there may be a dose-response issue that needs to be further examined, and that moderate intensity resistance training may not generate the stiffening effects on the arteries, which is often observed with high-intensity resistance training.

Our findings do not support those of Miyachi et $\mathrm{al}^{10}$ who reported that arterial compliance decreased after 2 months of resistance training. The participants were nonobese men aged 20 to 38 years who were trained 3 days per week performing 3 sets of 8 to 12 reps at $80 \%$ of $1-\mathrm{RM}$, which is a similar protocol to our study. The major difference between training protocols is the inclusion of lower and upper body resistance exercise, whereas our focus was on lower body exercises.

An unexpected finding of the current study was the increase in arterial compliance in $\mathrm{Cl}$ for the 2 groups, with a relative change of $9 \%$ and $13 \%$ for the training group and the control group, respectively. The changes observed in $\mathrm{C} 1$ are not believed to be because of measurement error since the coefficients of variation were less $(6.7 \%)$ than the observed relative changes with training. Physical activity outside the study was not monitored, which is a limitation (although they were instructed to continue their normal daily activity), and it is possible that if both groups engaged in more outdoor endurance type of activities as spring was approaching, this could have affected vessel compliance. Recreational physical activity has been associated with increased arterial compliance. ${ }^{1,35}$ 
Our study found no significant changes in C2 $(P>.05)$, which is in agreement with previous studies that found no differences in $\mathrm{C} 2$ between sedentary and resistance trained men ${ }^{10}$ and women. ${ }^{19}$ It has been suggested that changes in arterial compliance is more rapid in the central, more elastic arteries compared to the peripheral, more muscular arteries, ${ }^{36}$ and that may in part explain why we did not observe any changes in the small arteries in just 12 weeks of training. Perhaps, a training program of longer duration is necessary to detect any changes in the peripheral arteries.

\section{Conclusion}

In conclusion, 12 weeks of lower body resistance training did not decrease arterial compliance in our group of premenopausal women. Our findings suggest that lower body resistance training may not have the same negative effect on arterial compliance in women as often observed in men. Furthermore, if this is correct, this could be a good approach for postmenopausal women who want to preserve bone mass and muscle strength and at the same time maintain a healthy cardiovascular system.

\section{References}

1. Havlik RJ, Simmonsick EM, Sutton-Tyrrell K, et al, for the Health ABC Investigators. Association of physical activity and vascular stiffness in 70 to 79 year olds: the Health ABC Study. J Aging Phys Activity. 2003;11: 156-166.

2. Arnett DK, Evans GW, Riley WA. Arterial stiffness: a new cardiovascular risk factor? Am J Epidemiol. 1994;140: 669-682.

3. Howard G, Sharret AR, Heiss G. Carotid artery intimalmedial thickness distribution in general populations as evaluated by B-mode ultrasound. Stroke. 1993;24: 1297-1304.

4. Galetta F, Rossi M, Franzoni F, Credidio L, Vagheggini G. Atherosclerosis vascular damage in elderly athletes and sedentary people. Angiology. 1997;48: 623-628.

5. Jensen-Urstad K, Bouvier F, Jensen-Urstad M. Preserved vascular reactivity in elderly male athletes. Scand J Med Sci Sports. 1999;9:88-91.

6. Manzi S, Wasko MC. Inflammation-mediated rheumatic disease and atherosclerosis. Ann Rheum Dis. 2000;59: 321-325.
7. Van Doornum S, McColl G, Wicks IP. Accelerated atherosclerosis: an extraarticular feature of rheumatoid arthritis? Arthritis Rheum. 2002;46:862-873.

8. Seals D, Stevenson E, Jones P, DeSouza C, Tanaka H. Lack of age-associated elevations in 24-h systolic and pulse pressures in women who exercise regularly. Am J Physiol Heart Circ Physiol. 1999;277:H947-H955.

9. Kingwell BA. Large artery stiffness: implications for exercise capacity and cardiovascular risk. Clin Exp Pharmacol Physiol. 2002;29:214-217.

10. Miyachi M, Donato A, Yamamoto K, et al. Greater agerelated reductions in central arterial compliance in resistance-trained men. Hypertension. 2003;41:130-135.

11. Miyachi M, Kawano H, Sugawara J, et al. Unfavorable effects of resistance training on central arterial compliance. A randomized intervention study. Circulation. 2004;110: 2858-2863.

12. Fuchsjäger-Mayrl G, Pleiner J, Wiesinger GF, et al. Exercise training improves vascular endothelial function in patients with type 1 diabetes. Diabetes Care. 2002;25: 1795-1801.

13. Tanaka H, Dinenno FA, Monahan KD, Clevenger CM, DeSouza CA, Seals DR. Aging, habitual exercise, and dynamic arterial compliance. Circulation. 2000;102: 1270-1275.

14. Mattusch F, Dufaux B, Heine O, Mertens I, Rost R. Reduction of the plasma concentration of C-reactive protein following nine months of endurance training. Int J Sports Med. 2000;21:21-24.

15. Kullo IJ, Khaleghi M, Hensrud DD. Markers of inflammation are inversely associated with VO2 max in asymptomatic men. J Appl Physiol. 2007;102:1374-1379.

16. Kuo HK, Yen CJ, Chen JH, Yu YH, Bean JF. Association of cardiorespiratory fitness and levels of C-reactive protein: data from the National Health and Nutrition Examination Survey 1999-2002. Int J Cardiol. 2007;1 14: 28-33.

17. Kawano H, Tanaka H, Miyachi M. Resistance training and arterial compliance: keeping the benefits while minimizing the stiffening. J Hypertens. 2006;24:1753-1759.

18. Casey DP, Pierce GL, Howe KS, Mering MC, Braith RW. Effect of resistance training on arterial wave reflection and brachial artery reactivity in normotensive postmenopausal women. Eur J Appl Physiol. 2007;100: 403-408.

19. Cortez-Cooper MY, DeVan AE, Anton MM, et al. Effects of high intensity resistance training on arterial stiffness and wave reflection in women. Am J Hypertens. 2005;18: 930-934.

20. Williams MRI, Westerman RA, Kingwell BA, et al. Variations in endothelial function and arterial compliance during the menstrual cycle. J Clin Endocrinol Metab. 2001;86:5389-5395.

21. Lane KV, Prisant ME, Prisant LM. Noninvasive vascular compliance: short-term assessment of test repeatability. Am J Hypertens. 2000;13:191-192. 
22. Lehman ED. Pulsewave velocity as a marker of cardiovascular disease. Lancet. 1996;348:744.

23. Prisant LM, Pasi M, Jupin D, Prisant ME. Assessment of repeatability and correlates of arterial compliance. Blood Press Monit. 2002;7:231-235.

24. Prisant LM, Resnick LM, Hollenberg SM. Arterial elasticity among normotensive subjects and treated and untreated hypertensive subjects. Blood Press Monit. 2001;6:233-237.

25. Lehman ED, Riley WA, Clarkson P, Gosling RG. Noninvasive assessment of cardiovascular disease in diabetes mellitus. Lancet. 1997;350:14-19.

26. Taddei S, Galetta F, Virdis A, et al. Physical activity prevents age-related impairment in nitric oxide availability in elderly athletes. Circulation. 2000;25: 2896-2901.

27. Rakobowchuck M, McGowan CL, de Groot PC, Hartman JW, MacDonald MJ. Endothelial function of young healthy males following whole body resistance training. J Appl Physiol. 2005;98:2185-2190.

28. Rakobowchuk M, McGowan CL, de Groot PC, et al. Effect of whole body resistance training on arterial compliance in young men. Exp Physiol. 2005;90:645-651.

29. DeVan AE, Anton MM, Cook JN, Neidre DB, CortezCooper MY, Tanaka H. Acute effects of resistance exercise on arterial compliance. J Appl Physiol. 2005;98: 2287-2291.
30. Bertovic DA, Waddell TK, Gatzka CD, Cameron JD, Dart AM, Kingwell BA. Muscular strength training is associated with low arterial compliance and high pulse pressure. Hypertension. 1999;33:1385-1391.

31. Overend TJ, Versteegh TH, Thompson E, Birmingham TB, Vandervoort AA. Cardiovascular stress associated with concentric and eccentric isokinetic exercise in young and older adults. J Gerontol A Biol Sci Med Sci. 2000;55: B177-B182.

32. Okamoto T, Masuhara M, Ikuta K. Effects of eccentric and concentric resistance training on arterial stiffness. J Hum Hypertens. 2006;20:348-354.

33. Kingwell BA, Berry KL, Cameron JD, Jennings GL, Dart AM. Arterial compliance increases after moderate-intensity cycling. Am J Physiol Heart Circ Physiol. 1997;273:H2186-H2191.

34. Maeda S, Otsuki T, Lemitsu M, et al. Effects of leg resistance on arterial function in older men. Br J Sports Med. 2006;40:867-869.

35. Seals DR. Habitual exercise and the age-associated decline in large artery compliance. Exerc Sport Sci Rev. 2003;31:68-72.

36. Hayashi K, Sugawara J, Komine H, Maeda S, Yoko T. Effects of aerobic exercise training on the stiffness of central and peripheral arteries in middle-aged sedentary men. Jpn J Physiol. 2005;55:235-239.

For reprints and permission queries, please visit SAGE's Web site at http:/www.sagepub.com/journalsPermissions.nav 